Wojciech Rosa (Lublin)

\title{
STOCHASTIC APPROXIMATION PROCEDURE IN SEMI-MARKOV ENVIRONMENT APPLIED TO ALCOHOL CONSUMPTION MODEL
}

Abstract. In this paper, we consider a stochastic approximation procedure with semi-Markov switchings in an averaging scheme with a small parameter.

1. Introduction. Due to the wide use of stochastic diffusion problems, conditions for stability and control of such systems seem to be important. In [6] sufficient conditions for stability of stochastic systems via Lyapunov function properties are given and estimates of large deviations of linear diffusion systems are obtained. Problems of optimal control of diffusion processes described by stochastic differential equations with acceptable control are described in [20]. On the other hand, asymptotic behaviour is considered in [25] and [26].

For conditions of weak convergence of random processes in [11, 16, 12 the method of small parameter and a singular perturbation problem solution was used for the construction of the generator limiting process. This method is applied to schemes of averaging of diffusion approximation and to schemes of averaging of asymptotically small diffusion. In particular in [16] the cases of random evolution of Markov and semi-Markov switchings were examined.

Construction of semi-Markov processes and investigation of asymptotic properties of random processes with semi-Markov switchings are considered in [1, 2, 3, 4]. For these processes, weak convergence to the solution of ap-

2020 Mathematics Subject Classification: Primary 60F05, 60J60, 60J70; Secondary 97A40. Key words and phrases: stochastic approximation procedure, compensating operator, asymptotic normality of the stochastic procedure, small parameter, Markov and semiMarkov processes, alcohol consumption model.

Received 15 November 2018; revised 29 August 2019 and 23 February 2020.

Published online 11 May 2020. 
propriate partial differential equations and an averaging scheme of diffusion processes in diffusion approximation is presented in [3, 5].

In [19] asymptotic properties of semi-Markov processes with linearly perturbed operator maintainer a Markov process were analysed via the semigroup property. These results were then developed in [14]. A classification of solutions of a singular perturbation problem for random processes with the use of semi-Markov switchings is described in [16] and in [15] with the use of the compensating operator (see [27]). Using the compensating operator [10] one obtains sufficient conditions for convergence of a random evolution with semi-Markov switchings to the diffusion process in the averaging scheme (see also [13]).

The results of these studies have found various applications [8, 9, 18, 17].

In 21] convergence of stochastic procedures is established using properties of Lyapunov type functions. Stochastic Approximation Procedure (SAP) by a regression function with semi-Markov switchings was considered in [7].

2. Problem. In this paper, we consider a dynamical system with semiMarkov switchings using a small parameter. $x(t), t \geq 0$, is a semi-Markov process in the standard phase space $(X, \mathcal{E})$, generated by the renewal Markov process $x_{n}, \tau_{n}, n \geq 0$, defined by the semi-Markov kernel

$$
Q(t, x, B)=P(x, B) G_{x}(t),
$$

where the stochastic kernel

$$
P(x, B):=P\left\{x_{n+1} \in B \mid x_{n}=x\right\}, \quad B \in \mathcal{E},
$$

defines an embedded Markov chain $x_{n}=x\left(\tau_{n}\right)$ at the renewal moments,

$$
\tau_{n}=\sum_{k=1}^{n} \theta_{k}, \quad n \geq 0, \quad \tau_{0}=0,
$$

with intervals $\theta_{k+1}=\tau_{k+1}-\tau_{k}$ between the renewal moments. The $\theta_{n}$ are defined by the distribution functions

$$
G_{x}(t)=P\left\{\theta_{n+1} \leq t \mid x_{n}=x\right\}=: P\left\{\theta_{x} \leq t\right\} .
$$

Define

$$
g(x)=\int_{0}^{\infty} \bar{G}_{x}(d s), \quad \bar{G}_{x}(s)=1-G_{x}(s) .
$$

The semi-Markov process is defined by

$$
x(t)=x_{\nu(t)}, \quad t \geq 0,
$$

where the counting process $\nu(t)$ is defined by

$$
\nu(t):=\max \left\{n: \tau_{n} \leq t\right\}, \quad t \geq 0 .
$$


We shall assume that the semi-Markov process $x(t), t \geq 0$, is regular (the probability of reaching any state is positive) and uniformly ergodic [16, p. 33] with stationary distribution $\pi(B), B \in \mathcal{E}$ :

$$
\pi(d x)=\rho(d x) g(x) / m, \quad \text { where } \quad m=\int_{X} g(x) \rho(d x) .
$$

Here $\rho(B), B \in \mathcal{E}$, is a stationary distribution of the embedded Markov chain $\left(x_{n}\right)$. Note that the process $x(t)$ has a generator $\mathbf{Q}$,

$$
\mathbf{Q} \phi(x)=\frac{1}{g(x)} \int_{X} P(x, d y)[\phi(y)-\phi(x)],
$$

which acts in the Banach space $\mathbb{B}$ of all bounded real-valued measurable functions on $X$, with the sup-norm $\|\phi\|=\sup _{x \in X}|\phi(x)|$ for $\phi \in \mathbb{B}$. We have

$$
\mathbb{B}=N_{\mathbf{Q}} \oplus R_{\mathbf{Q}}
$$

where $N_{\mathbf{Q}}:=\{\phi: \mathbf{Q} \phi=0\}$ and $R_{\mathbf{Q}}:=\{\mathbf{Q} \psi: \psi \in \mathbb{B}\}$. Given $\mathbf{Q}$, we can define the potential operator or simply the potential of $\mathbf{Q}$ by

$$
\mathbf{R}_{0}=\Pi-(\mathbf{Q}+\Pi)^{-1} \quad \text { where } \quad \Pi \phi(x):=\int_{X} \pi(d x) \phi(x) .
$$

SAP for a diffusion process $u^{\varepsilon}(t) \in \mathbb{R}^{d}$ in an averaging scheme with a small parameter $\varepsilon>0$ is defined by a stochastic differential equation

$$
d u^{\varepsilon}(t)=a(t)\left[C\left(u^{\varepsilon}(t) ; x\left(\frac{t}{\varepsilon}\right)\right) d t+\sigma\left(u^{\varepsilon}(t) ; x\left(\frac{t}{\varepsilon}\right)\right) d w(t)\right]
$$

where

- $u^{\varepsilon}(t), t \geq 0$, is a random evolution in a diffusion process [16, 2, 15, 27];

- $x(t), t \geq 0$, is a semi-Markov process [16, 1, 19, 14];

- $w(t)$ is the Wiener process [25, 26, 11],

- $a(t)$ satisfies $\int_{t_{0}}^{\infty} a(t) d t=\infty, \int_{t_{0}}^{\infty} a^{2}(t) d t<\infty$.

The semigroup $\mathbf{T}_{x}(t), t \geq 0, x \in X$, associated to the system

$$
d u_{x}(t)=a(t)\left[C\left(u_{x}(t) ; x\right) d t+\sigma\left(u_{x}(t) ; x\right) d w(t)\right], \quad u_{x}(0)=u,
$$

is defined by

$$
\mathbf{T}_{x}(t) \phi(u)=\phi\left(u_{x}(t, u)\right)
$$

where

$$
u_{x}(t, u):=u_{x}(t), \quad u_{x}(0)=u .
$$

Notice that $u_{x}(t+s, u)=u_{x}\left(s, u_{x}(t, u)\right)$, which is the semigroup property for the trajectories $u_{x}(t, u)$ [16, p. 44].

The generating operator $\mathbf{A}_{x}(t)$ of the semigroup $\mathbf{T}_{x}(t)$ is defined by

$$
\mathbf{A}_{x}(t) \phi(u)=a(t) C(u, x) \phi^{\prime}(u)+a^{2}(t) \frac{1}{2} \sigma^{2}(u, x) \phi^{\prime \prime}(u),
$$


where $\phi(\cdot) \in C^{2}\left(\mathbb{R}^{d}\right)$ and $a(\cdot) \in C^{1}(\mathbb{R})$. Note that a solution of 3 exists when the following conditions are satisfied:

$$
\begin{aligned}
& \left\|C\left(u_{1}, x\right)-C\left(u_{2}, x\right)\right\|+\left\|\sigma\left(u_{1}, x\right)-\sigma\left(u_{2}, x\right)\right\|<l\left(\left\|u_{1}-u_{2}\right\|\right), \quad \forall x \in X, \\
& \|C(u, x)\|+\|\sigma(u, x)\|<l(1+\|u\|), \quad \forall x \in X,
\end{aligned}
$$

for some $l>0$ [21, Chapter 3, Sec. 4].

3. Main result. The main result of the paper can be formulated as follows:

Theorem 3.1. Let $C(\cdot, x) \in C^{3}\left(\mathbb{R}^{d}\right)$ for all $x \in X$ and let $V(u)$ be the Lyapunov function for the dynamical system $\frac{d u}{d t}=C(u)$, where $C(u)=$ $\int_{X} C(u, x) \pi(d x)$, which satisfies the following conditions:

(C1) $C(u) V^{\prime}(u) \leq-c_{0} V(u), c_{0}>0$,

(C2) $\left|R_{0} C(u, x) V^{\prime}(u)\right| \leq c_{1}(1+V(u))$,

(C3) $\left|C(u, x) R_{0}\left[C(u, x) V^{\prime}(u)\right]^{\prime}\right| \leq c_{2}(1+V(u))$,

(C4) $\left|C(u, x)\left[C(u, x)\left[C(u, x) V^{\prime}(u)\right]^{\prime}\right]^{\prime}\right| \leq c_{3}(1+V(u))$,

(C5) (Cramer condition) $\sup _{x \in X} \int_{0}^{\infty} e^{h t} G_{x}(t) d t \leq H<\infty, h>0$,

(C6) $\int_{0}^{\infty} a(t) d t=\infty, \int_{0}^{\infty} a^{2}(t) d t<\infty$,

(C7) $b_{1}^{\varepsilon}(t, s)=\left|\frac{a(t+\varepsilon s)}{a(t)}\right| \leq A_{1}<\infty, a(\cdot) \in C^{1}(\mathbb{R})$ and $b_{2}^{\varepsilon}(t, s)=\left|\frac{a^{\prime}(t+\varepsilon s)}{a(t)}\right|<$ $A_{2}<\infty$.

Then the solution $u^{\varepsilon}(t), t \geq 0$, of $(2)$ converges to some point $u^{*} \in \mathbb{R}$ almost surely.

We introduce an advanced Markov renewal process (MRP) (see [16]), using the given sequence:

$$
u_{n}^{\varepsilon}=u^{\varepsilon}\left(\tau_{n}^{\varepsilon}\right), \quad x_{n}^{\varepsilon}=x^{\varepsilon}\left(\tau_{n}^{\varepsilon}\right), \quad \tau_{n}^{\varepsilon}=\varepsilon \tau_{n},
$$

where $\tau_{n}=\sum_{k=1}^{n} \theta_{k}, n \geq 0, \tau_{0}=0$ are the renewal times of the semi-Markov process $x(t), t \geq 0$, determined by the distribution function of the time spent in state $x$.

Definition 1 ([16, 10]). The compensating operator of the advanced MRP (6) is defined by

$$
\begin{aligned}
& \mathbf{L}_{t}^{\varepsilon}(x) \phi(u, x) \\
& \quad=\varepsilon^{-1}\left[E\left\{\phi\left(u_{n+1}^{\varepsilon}, x_{n+1}^{\varepsilon}\right) \mid u_{n}^{\varepsilon}=u, x_{n}^{\varepsilon}=x, \tau_{n}^{\varepsilon}=t\right\}-\phi(u, x)\right] / g(x) .
\end{aligned}
$$

To simplify notation we let

$$
q(x)=\frac{1}{g(x)} \quad \text { and } \quad \mathbf{P} \phi(u, x)=\int_{X} \phi(u, y) P(x, d y) .
$$


We have

Lemma 3.2. The compensating operator $\mathbf{L}_{t}^{\varepsilon}(x)$ is of the following form:

$$
\mathbf{L}_{t}^{\varepsilon}(x) \phi(u, x)=\varepsilon^{-1} \mathbf{Q} \phi(u, x)+\varepsilon^{-1}\left[\mathbf{G}_{t}^{\varepsilon}(x)-I\right] \mathbf{Q}_{0} \phi(u, x),
$$

where

$$
\mathbf{G}_{t}^{\varepsilon}(x)=\int_{0}^{\infty} G_{x}(d s) \mathbf{T}_{x}(t+\varepsilon s), \quad \mathbf{Q}_{0} \phi(x)=\frac{1}{g(x)} \mathbf{P} \phi(u, x) .
$$

Proof. From [16, 27, 13] we have

$$
\begin{aligned}
E\left[\phi\left(u_{n+1}^{\varepsilon}, x_{n+1}^{\varepsilon}\right) \mid u_{n}^{\varepsilon}=u, x_{n}^{\varepsilon}=x,\right. & \left.\tau_{n}^{\varepsilon}=t\right]=E_{u, x, t} \phi\left(u_{n+1}^{\varepsilon}, x_{n+1}^{\varepsilon}\right) \\
& =\int_{0}^{\infty} G_{x}(d s) \mathbf{T}_{x}(t+\varepsilon s) \int_{X} P(x, d y) \phi(u, y) .
\end{aligned}
$$

Hence

$$
\begin{aligned}
\mathbf{L}_{t}^{\varepsilon}(x) \phi(u, x) & \\
= & \varepsilon^{-1} q(x)\left[\int_{0}^{\infty} G_{x}(d s) \mathbf{T}_{x}(t+\varepsilon s) \int_{X} P(x, d y) \phi(u, y)-\phi(u, x)\right] \\
= & \varepsilon^{-1} q(x) \int_{X} P(x, d y)[\phi(u, y)-\phi(u, x)] \\
& +\varepsilon^{-1} q(x) \int_{0}^{\infty} G_{x}(d s)\left[\mathbf{T}_{x}(t+\varepsilon s)-I\right] \int_{X} P(x, d y) \phi(u, y) .
\end{aligned}
$$

Thus we obtain (8).

LEMma 3.3. The compensating operator $\mathbf{L}_{t}^{\varepsilon}(x)$ has the asymptotic representations

(9) $\mathbf{L}_{t}^{\varepsilon}(x) \phi(u, x)=\varepsilon^{-1} \mathbf{Q} \phi(u, x)+q(x) \theta_{1}^{\varepsilon}(x) \mathbf{P} \phi(u, x)$,

(10) $\mathbf{L}_{t}^{\varepsilon}(x) \phi(u, x)=\varepsilon^{-1} \mathbf{Q} \phi(u, x)+\mathbf{A}_{x}(t) \mathbf{P} \phi(u, x)+\varepsilon^{2} a^{2}(t) \theta_{2}^{\varepsilon}(x) \mathbf{P} \phi(u, x)$, where

$$
\begin{aligned}
& \theta_{1}^{\varepsilon}(x)=\int_{0}^{\infty} \bar{G}_{x}(s) \mathbf{A}_{x}(t+\varepsilon s) \mathbf{T}_{x}(t+\varepsilon s) d s \\
& \theta_{2}^{\varepsilon}(x)=q(x) \int_{0}^{\infty} \bar{G}_{x}^{(2)}(s) \widetilde{\mathbf{A}}_{x}^{1}(t+\varepsilon s) \widetilde{\mathbf{A}}_{x}(t+\varepsilon s) \mathbf{T}_{x}(t+\varepsilon s) d s
\end{aligned}
$$

where

$$
\begin{aligned}
\widetilde{\mathbf{A}}_{x}^{1}(t+\varepsilon s) \phi(u) & =b_{2}^{\varepsilon}(t, s) C(u, x) \phi^{\prime}(u)+a(t+\varepsilon s) b_{2}^{\varepsilon}(t, s) \sigma^{2}(u, x) \phi^{\prime \prime}(u) \\
& =\frac{1}{a(t)} \mathbf{A}_{x}^{\prime}(t+\varepsilon s) \phi(u)
\end{aligned}
$$




$$
\begin{aligned}
\widetilde{\mathbf{A}}_{x}(t+\varepsilon s) \phi(u) & =b_{1}^{\varepsilon}(t, s) C(u, x) \phi^{\prime}(u)+\frac{1}{2} a(t+\varepsilon s) b_{1}^{\varepsilon}(t, s) \sigma^{2}(u, x) \phi^{\prime \prime}(u) \\
& =\frac{1}{a(t)} \mathbf{A}_{x}(t+\varepsilon s) \phi(u), \\
b_{1}^{\varepsilon}(t, s) & =\frac{a(t+\varepsilon s)}{a(t)}, \quad b_{2}^{\varepsilon}(t, s)=\frac{a^{\prime}(t+\varepsilon s)}{a(t)}
\end{aligned}
$$

Proof. For the semigroup $\mathbf{T}_{x}(t+\varepsilon s), t \geq 0, x \in X$, we have,

$$
d \mathbf{T}_{x}(t+\varepsilon s)=\varepsilon \mathbf{A}_{x}(t+\varepsilon s) \mathbf{T}_{x}(t+\varepsilon s) d s .
$$

Integrating by parts we obtain (see (8))

$$
\begin{aligned}
\mathbf{G}_{t}^{\varepsilon}(x)-I & =\int_{0}^{\infty} G_{x}(d s)\left[\mathbf{T}_{x}(t+\varepsilon s)-I\right] \\
& =-\left.\bar{G}_{x}(s)\left[\mathbf{T}_{x}(t+\varepsilon s)-I\right]\right|_{0} ^{\infty}+\varepsilon \int_{0}^{\infty} \bar{G}_{x}(s) \mathbf{A}_{x}(t+\varepsilon s) \mathbf{T}_{x}(t+\varepsilon s) d s .
\end{aligned}
$$

Given the Cramer condition we have $\lim _{s \rightarrow \infty} \bar{G}_{x}(s)=0$. Moreover $T_{x}(t)=I$, so

$$
\mathbf{G}_{t}^{\varepsilon}(x)-I=\varepsilon \int_{0}^{\infty} \bar{G}_{x}(s) \mathbf{A}_{x}(t+\varepsilon s) \mathbf{T}_{x}(t+\varepsilon s) d s=\varepsilon \theta_{1}^{\varepsilon}(x) .
$$

Hence we obtain (9).

For

$$
\mathbf{G}_{t, 1}^{\varepsilon}(x)=\int_{0}^{\infty} \bar{G}_{x}(s) \mathbf{A}_{x}(t+\varepsilon s) \mathbf{T}_{x}(t+\varepsilon s) d s
$$

integrating by parts using the substitutions $u=\mathbf{A}_{x}(t+\varepsilon s) \mathbf{T}_{x}(t+\varepsilon s)$ and $d v=\bar{G}_{x}(d s)$ with $d u=\left(\mathbf{A}_{x}(t+\varepsilon s) \mathbf{T}_{x}(t+\varepsilon s)\right)^{\prime} d s$ and $v=-\bar{G}_{x}^{(2)}(s)$ we get

$$
\begin{aligned}
\mathbf{G}_{t, 1}^{\varepsilon}(s)= & \int_{0}^{\infty} \bar{G}_{x}(s) \mathbf{A}_{x}(t+\varepsilon s) \mathbf{T}_{x}(t+\varepsilon s) d s \\
= & -\left.\mathbf{A}_{x}(t+\varepsilon s) \mathbf{T}_{x}(t+\varepsilon s) \cdot \bar{G}_{x}^{(2)}(s)\right|_{0} ^{\infty} \\
& +\varepsilon \int_{0}^{\infty}\left(\mathbf{A}_{x}(t+\varepsilon s) \mathbf{T}_{x}(t+\varepsilon s)\right)^{\prime} \cdot \bar{G}_{x}^{(2)}(s) d s \\
= & g(x) \mathbf{A}_{x}(t) I+\varepsilon^{2} \int_{0}^{\infty} \mathbf{A}_{x}^{\prime}(t+\varepsilon s) \mathbf{A}_{x}(t+\varepsilon s) \mathbf{T}_{x}(t+\varepsilon s) \cdot \bar{G}_{x}^{(2)}(s) d s \\
= & g(x) \mathbf{A}_{x}(t) I+\varepsilon^{2} a^{2}(t) g(x) \theta_{2}^{\varepsilon}(x),
\end{aligned}
$$

where

$$
\bar{G}_{x}^{(2)}(t)=\int_{t}^{\infty} \bar{G}_{x}(s) d s .
$$

Substituting this result into (9), we obtain 10 . 
Lemma 3.4. The compensating operator $\mathbf{L}_{t}^{\varepsilon}(x)$ satisfies, with $\phi^{\varepsilon}(u, x)=$ $\phi(u)+\varepsilon \phi_{1}(u, x)$,

$$
\mathbf{L}_{t}^{\varepsilon}(x) \phi^{\varepsilon}(u, x)=\mathbf{L}_{t} \phi(u, x)+\varepsilon \theta_{t}^{\varepsilon}(x) \phi(u)
$$

where $\theta_{t}^{\varepsilon}(x) \phi(u)=q(x) \theta_{1}(x) \mathbf{P} \mathbf{R}_{0} \widetilde{\mathbf{L}}_{t}(x) \phi(u)+\varepsilon a^{2}(t) \theta_{2}^{\varepsilon}(x) \phi(u), \widetilde{\mathbf{L}}_{t}(x)=$ $\mathbf{A}_{x}(t)-\mathbf{L}_{t}$ and $\phi(\cdot) \in C^{3}(\mathbb{R})$.

Proof. We have

$\mathbf{L}_{t}^{\varepsilon}(x)\left[\phi(u)+\varepsilon \phi_{1}(u, x)\right]=$

$\varepsilon^{-1} \mathbf{Q} \phi(u)+\mathbf{A}_{x}(t) \phi(u)+\mathbf{Q} \phi_{1}(u, x)+\varepsilon q(x) \theta_{1}^{\varepsilon}(x) \mathbf{P} \phi_{1}(u, x)+\varepsilon a^{2}(t) \theta_{2}^{\varepsilon}(x) \mathbf{P} \phi(u)$.

Now

$$
\mathbf{L}_{t} \phi(u)=\Pi \mathbf{A}_{x}(t) \Pi \phi(u)=a(t) C(u) \phi^{\prime}(u)+\frac{a^{2}(t) \sigma^{2}(u)}{2} \phi^{\prime \prime}(u)
$$

where

$$
C(u)=\int_{X} C(u, x) \pi(d x), \quad \sigma^{2}(u)=\int_{X} \sigma^{2}(u, x) \pi(d x) .
$$

From $\phi(\cdot) \in N_{\mathbf{Q}}$ we have

$$
\mathbf{A}_{x}(t) \phi(u)+\mathbf{Q} \phi_{1}(u, x)=\mathbf{L}_{t} \phi(u),
$$

Hence

$$
\mathbf{Q} \phi_{1}(u, x)=\left(\mathbf{A}_{x}(t)-\mathbf{L}_{t}\right) \phi(u)=\widetilde{\mathbf{L}}_{t}(x) \phi(u) .
$$

Using this result we obtain

$$
\phi_{1}(u, x)=\mathbf{R}_{0} \widetilde{\mathbf{L}}_{t}(x) \phi(u) .
$$

Finally,

$$
\mathbf{L}_{t}^{\varepsilon}(x) \phi^{\varepsilon}(u, x)=\mathbf{L}_{t} \phi(u)+\varepsilon \theta_{t}^{\varepsilon}(x) \phi(u)
$$

where

$$
\theta_{t}^{\varepsilon}(x)=q(x) \theta_{1}(x) \mathbf{P} \mathbf{R}_{0} \widetilde{\mathbf{L}}_{t}(x)+a^{2}(t) \theta_{2}^{\varepsilon}(x) .
$$

Consider a Lyapunov function $V(u)$ for the equation

$$
\frac{d u}{d t}=C(u) \text {. }
$$

The next lemma follows immediately from the previous one:

Corollary 3.5. For the Lyapunov function $V^{\varepsilon}(u, x)=V(u)+\varepsilon V_{1}(u, x)$ we have

$$
\mathbf{L}_{t}^{\varepsilon}(x) V^{\varepsilon}(u, x)=\mathbf{L}_{t} V(u)+\varepsilon \theta_{t}^{\varepsilon}(x) V(u) .
$$

Finally, we are ready to prove our main Theorem 3.1 .

Proof of Theorem 3.1. Using conditions (C4)-(C7) we have

$$
\left|\theta_{t}^{\varepsilon}(x) V(u)\right| \leq A_{3}(1+V(u))<\infty .
$$


Next, using conditions $(\mathrm{C} 1)-(\mathrm{C} 3)$ we find that

$$
\mathbf{L}_{t} V(u) \leq-c_{0} a(t) V(u)+a^{2}(t) c_{1}(1+V(u)) .
$$

Now from the Korolyuk theorem (see Theorem 6.1 or [16]) and the NevelsonHasminskii theorem (see Theorem 6.2 or [21]) we deduce that

$$
P\left(\lim _{\varepsilon \rightarrow 0} u^{\varepsilon}(t)=u^{\star}\right)=1,
$$

which completes the proof.

4. Application. This result can be used in controlling the process in an averaging scheme with semi-Markov switchings [22].

A mathematical model for alcohol consumption is considered [23]. In [24], an equilibrium point was obtained in the case of deterministic values of coefficients in the form

$$
\left\{\begin{aligned}
a^{\prime} & =\mu+\gamma-\gamma m+\beta a^{2}-a\left[\beta+\mu+\gamma-\left(d-d_{A}\right)(1-a)\right], \\
m^{\prime} & =\beta a-\beta a^{2}-m\left[\alpha+\mu+a\left(d-d_{A}\right)\right],
\end{aligned}\right.
$$

where $\alpha=0.000110247$ (rate at which a nonrisk consumer moves to the risk consumption subpopulation), $\beta=0.0284534$ (transmission rate due to social pressure to increase alcohol consumption: family, friends, marketing, TV, etc.), $\mu=0.01$ (birth rate in Spain), $d_{A}=0.008$ (death rate in Spain), $d=$ 0.009 (augmented death rate due to alcohol consumption), $\gamma=0.00144$ (rate at which a risk consumer becomes a nonconsumer), $a(t)$ is the rate of nonconsumers, and $m(t)$ the rate of non-risk consumers. It is the same model as presented in [23], but without delay (assuming $I\left(a_{t}\right) \approx a_{t}$ ).

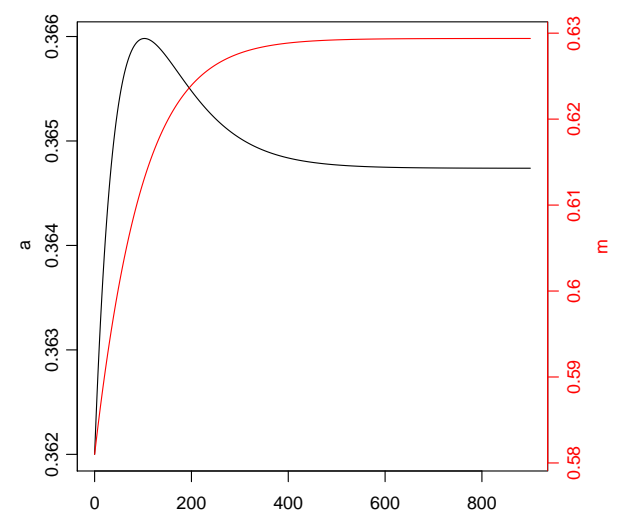

The solution of system (11) converges to the equilibrium point: $a^{*}=$ $0.3647389407, m^{*}=0.6293831151$ (see [23]). We propose below a modification of the above system considering a semi-Markov model with switchings. More precisely, we consider two modifications studied in the following subsections. 
4.1. Model with stochastic drift. Consider the model

$$
\left\{\begin{aligned}
a^{\prime}= & \mu+\gamma-\gamma m+\beta a^{2}-a\left[\beta+\mu+\gamma-\left(d-d_{A}\right)(1-a)\right] \\
& +\sigma_{1}\left(a-a^{\star}\right) w_{1}^{\prime}, \\
m^{\prime}= & \beta a-\beta a^{2}-m\left[\alpha+\mu+a\left(d-d_{A}\right)\right]+\sigma_{2}\left(m-m^{\star}\right) w_{2}^{\prime},
\end{aligned}\right.
$$

where $\alpha=0.000110247, \beta=0.0284534, \mu=0.01, d_{A}=0.008, d=0.009$, $\gamma=0.00144, a_{0}=0.362, m_{0}=0.581, a(t)$ is the nonconsumers rate, and $m(t)$ the rate of nonrisk consumers. It is the same model as in [24], $I\left(a_{t}\right) \approx a_{t}$. Here $w_{1}^{\prime}, w_{2}^{\prime}$ denote mutually independent standard Wiener processes.

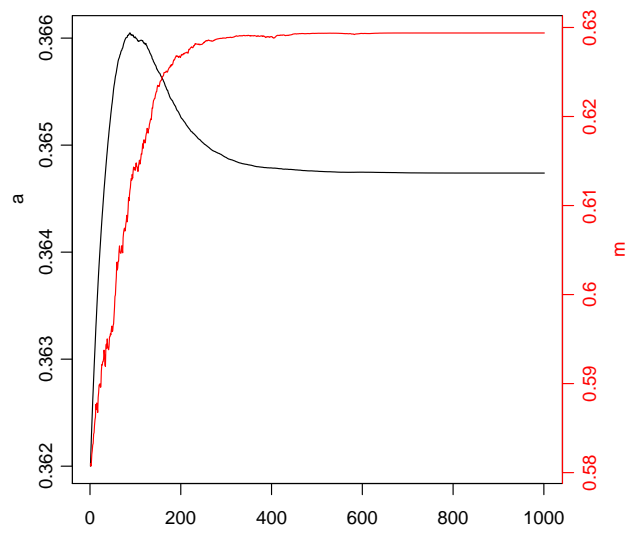

The solution of 12 converges to the equilibrium point $a^{*}=0.3647389407$, $m^{*}=0.6293831151$ [24].

4.2. Stochastic approximation procedure applied to stochastic drift with semi-Markov switchings. The next model involves nonconstant coefficients. In this case, we use a stochastic approximation procedure for a diffusion process with semi-Markov switchings,

$$
\left\{\begin{aligned}
a^{\prime}= & c\left[\mu(X)+\gamma-\gamma m+\beta a^{2}-a\left[\beta+\mu(X)+\gamma-\left(d-d_{A}\right)(1-a)\right]\right. \\
& \left.+\sigma_{1}\left(a-a^{\star}\right) w_{1}^{\prime}\right] \\
m^{\prime}= & c\left[\beta a-\beta a^{2}-m\left[\alpha+\mu(X)+a\left(d-d_{A}\right)\right]+\sigma_{2}\left(m-m^{\star}\right) w_{2}^{\prime}\right]
\end{aligned}\right.
$$

where $\alpha=0.000110247, \beta=0.0284534, \mu=0.01, d_{A}=0.008, d=0.009$, $\gamma=0.00144, a_{0}=0.362, m_{0}=0.581, a(t)$ is the rate of nonconsumers, and $m(t)$ the rate of nonrisk consumers. It is the same model as in [24, $I\left(a_{t}\right) \approx a_{t}, a^{\star}=0.3647389407, m^{\star}=0.6293831151$ (the solution of the simple model (12). Here $w_{1}^{\prime}, w_{2}^{\prime}$ are mutually independent standard Wiener processes and

$$
\mu(X)=\mu+X
$$


where $X$ is a semi-Markov process with states \pm 0.005 , and with $G_{x}(t) \sim$ unif $[0,50] . G_{x}(t)$ could have another distribution, but should satisfy the Cramer condition (see (C6) of Theorem 3.1).

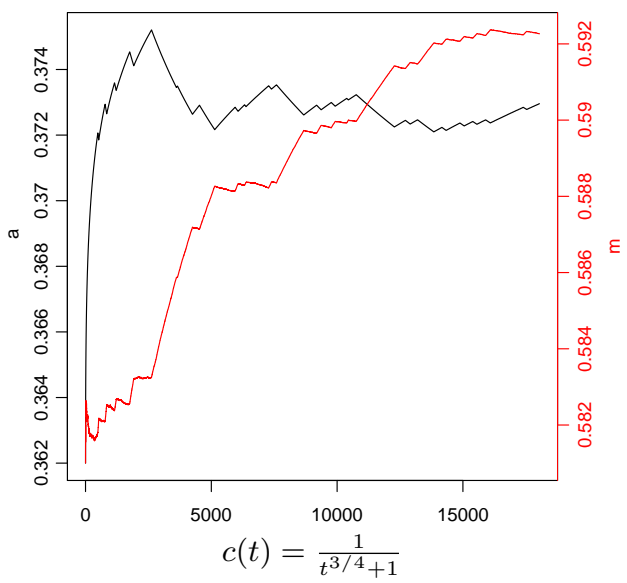

5. Conclusion. Considering the seventh condition of the theorem $\left(\int_{0}^{\infty} c(t) d t=\infty, \int_{0}^{\infty} c^{2}(t) d t<\infty\right)$, the following control functions are available:

$$
c(t)=\frac{1}{t^{\alpha}+1}
$$

where $\alpha \in(1 / 2,1]$. In our example, when $\alpha=1$ we get a slight local variation but slow convergence to the solution (figure (b)). In contrast, when $\alpha=1 / 2$, we get faster convergence to the solution, but more local variations (figure (a)). The selection of the appropriate $\alpha$ can be the subject of a separate study; in our opinion, it is reasonable to take $\alpha=3 / 4$, which combines the advantages and disadvantages of extreme values in this context.

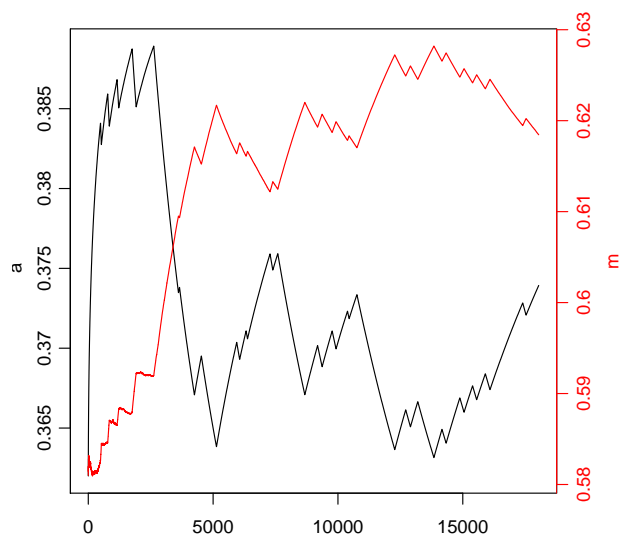

(a) $c(t)=\frac{1}{\sqrt{t}+1}$

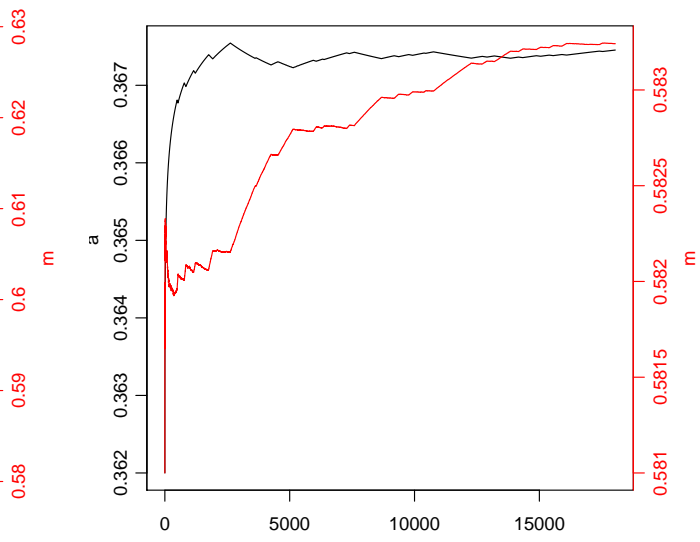

(b) $c(t)=\frac{1}{t+1}$ 


\section{Appendix}

Theorem 6.1 (Pattern limit theorem [16, p. 202]). Suppose the following conditions hold:

(D1) The family of stochastic processes $\xi^{\varepsilon}(t), t \geq 0, \varepsilon \geq 0$, is relatively compact.

(D2) There exists a family of test functions $\phi^{\varepsilon}(\cdot, \cdot)$ in $C_{0}^{3}(\mathbb{R} \times E)$ such that $\lim _{\varepsilon \rightarrow 0} \phi^{\varepsilon}(u, x)=\phi(u) \quad$ uniformly in $u, x$.

(D3) We have

$$
\lim _{\varepsilon \rightarrow 0} \mathbf{L}_{t}^{\varepsilon} \phi^{\varepsilon}(u, x)=\mathbf{L}_{t} \phi(u) \quad \text { uniformly on } u, x .
$$

The family of functions $\mathbf{L}_{t}^{\varepsilon} \phi^{\varepsilon}, \varepsilon>0$, is uniformly bounded, and $\mathbf{L}_{t}^{\varepsilon} \phi^{\varepsilon}$ and $\mathbf{L}_{t} \phi$ belong to $C\left(\mathbb{R}^{d} \times E\right)$.

(D4) The convergence of the initial values holds, that is,

$$
\xi^{\varepsilon}(0) \rightarrow \xi(0), \quad \varepsilon \rightarrow 0
$$

and

$$
\sup _{\varepsilon>0} \mathbb{E}\left|\xi^{\varepsilon}(0)\right| \leq C<+\infty .
$$

Then we have the weak convergence

$$
\xi^{\varepsilon}(t) \rightarrow \xi(t), \quad \varepsilon \rightarrow 0 .
$$

The limit process $\xi(t), t \geq 0$, is given by the solution of

$$
\frac{d}{d t} \phi(\xi(t))=\mathbf{L}_{t} \phi(\xi)
$$

We used conditions (D2) and (D3) in (3.4). Condition (D1) has been proved in [27.

Let

$$
\mathbf{L}_{t} \phi(u)=a(t) C(u) \phi^{\prime}(u)+\frac{1}{2} a^{2}(t) \sigma^{2}(u) \phi^{\prime \prime}(u),
$$

where $\phi(\cdot) \in C^{2}\left(\mathbb{R}^{d}\right)$.

Theorem 6.2 ([21, Chapter 3, formula (8.5)]). Let $V(u)$ be a Lyapunov function such that

$$
V(u) \rightarrow \infty, \quad|u| \rightarrow \infty,
$$

and

$$
\mathbf{L}_{t} V(u) \leq-a(t) c_{0} V(u)+c_{1} a^{2}(u)(1+V(u))
$$

where $\int_{t_{0}}^{\infty} a(t) d t=\infty$ and $\int_{t_{1}}^{\infty} a^{2}(t) d t<\infty$, and $c_{0}>0, c_{1}>0$. Then

$$
P\left(\lim _{t \rightarrow \infty} u(t)=u^{\star}\right)=1,
$$

where $C\left(u^{\star}\right)=0$. 
W. Rosa

\section{References}

[1] V. V. Anisimov, Limit theorems for switching processes and their applications, Cybernetics 14 (1978), 917-929.

[2] V. V. Anisimov, Limit theorems for switching processes, Theory Probab. Math. Statist. 37 (1988), 1-5.

[3] V. V. Anisimov, Switching processes: Averaging principle, diffusion approximation and applications, Acta Applicandae Math. 40 (1995), 95-141.

[4] V. V. Anisimov, Averaging methods for transient regimes in overloading retrial queuing systems, Math. Comput. Modelling 30 (1999), 65-78.

[5] V. V. Anisimov, Switching Processes in Queueing Models, Wiley, 2008.

[6] G. Blankenship and G. Papanicolaou, Stability and control of stochastic systems with wide band noise disturbances, SIAM J. Appl. Math. 34 (1978), 437-476.

[7] Y. M. Chabanyuk, Continuous procedure of stochastic approximation in a semi-Markov medium, Ukrainian Math. J. 56 (2004), 862-872.

[8] Y. M. Chabanyuk, Continuous stochastic approximation with semi-Markov switchings in the diffusion approximation scheme, Cybernetics and Systems Anal. 43 (2007), 605-612.

[9] Y. M. Chabanyuk, Convergence of a jump procedure in a semi-Markov environment in a diffusion approximation scheme, Cybernetics and Systems Anal. 43 (2007), 866-875.

[10] Y. M. Chabanyuk, Stability of a dynamical system with semi-Markov switchings under conditions of diffusion approximation, Ukrainian Math. J. 59 (2007), 1441-1452.

[11] V. S. Korolyuk, Stability of stochastic systems in the diffusion-approximation scheme, Ukrainian Math. J. 50 (1998), 40-54.

[12] V. S. Korolyuk, Problem of large deviations for Markov random evolutions with independent increments in the scheme of asymptotically small diffusion, Ukrainian Math. J. 62 (2010), 739-747.

[13] V. S. Korolyuk and Y. M. Chabanyuk, Stability of a dynamical system with semi-Markov switchings under conditions of stability of the averaged system, Ukrainian Math. J. 54 (2002), 239-252.

[14] V. S. Korolyuk and V. V. Korolyuk, Stochastic Models of Systems, Kluwer, Dordrecht, 1999.

[15] V. S. Korolyuk, V. V. Korolyuk and N. Limnios, Queueing systems with semi-Markov flow in average and diffusion approximation schemes, Methodol. Comput. Appl. Probab. 11 (2009), 201-209.

[16] V. S. Korolyuk and N. Limnios, Stochastic Systems in Merging Phase Space, World Sci., Singapore, 2005.

[17] V. S. Korolyuk, N. Limnios, and I. V. Samoilenko, Poisson approximation of recurrent process with locally independent increments and semi-Markov switching-toward application in reliability, in: Advances in Degradation Modeling, Birkhäuser, 2010, $105-116$.

[18] V. S. Korolyuk, N. Limnios and I. V. Samoilenko, Poisson approximation of impulsive recurrent process with semi-Markov switching, Stochastic Anal. Appl. 29 (2011), $769-778$.

[19] V. S. Korolyuk and A. V. Swishchuk, Semi-Markov Random Evolutions, Kluwer, Dordrecht, 1995.

[20] H. J. Kushner, Optimality conditions for the average cost per unit time problem with a diffusion model, SIAM J. Control Optimization 16 (1978), 330-346.

[21] M. B. Nevelson and R. Z. Hasminskii, Stochastic Approximation and Recursive Estimation, Transl. Math. Monogr. 47, Amer. Math. Soc., 1973. 
[22] A. V. Nikitin and U. T. Khimka, Asymptotics of normalized control with Markov switchings, Ukrainian Math. J. 68 (2017), 1252-1262.

[23] F.-J. Santonja, E. Sánchez, M. Rubio and J.-L. Morera, Alcohol consumption in Spain and its economic cost: A mathematical modeling approach, Math. Computer Modelling 52 (2010), 999-1003.

[24] F.-J. Santonja and L. Shaikhet, Analysing social epidemics by delayed stochastic models, Discrete Dynam. Nature Soc. 2012, art. 530472, 13 pp.

[25] A. V. Skorokhod, Asymptotic Methods in the Theory of Stochastic Differential Equations, Amer. Math. Soc., 1989.

[26] D. W. Stroock and S. R. S. Varadhan, Multidimensional Diffusion Processes, Springer, Berlin, 1979.

[27] M. N. Sviridenko, Martingale approach to limit theorems for semi-Markov processes, Theor. Probab. Appl. 34 (1989), 540-545.

Wojciech Rosa

Department of Applied Mathematics

Lublin University of Technology

20-618 Lublin, Poland

E-mail: w.rosa@pollub.pl 
